\title{
Determinação de épocas de semeadura do feijão caupi no Recôncavo Baiano através do modelo CROPGRO
}

\author{
Aristides F. Lima Filho', Mauricio A. Coelho Filho ${ }^{2}$ \& Alexandre B. Heinemann ${ }^{3}$
}

\begin{abstract}
RESUMO
Neste estudo objetivou-se simular épocas de semeadura para o feijão caupi (Vigna unguiculata (L.) walp.) em Cruz das Almas, no Recôncavo Baiano, através do modelo CROPGRO-cowpea, calibrado e validado por meio de conjunto de dados provenientes de experimentos de campo. Dois cenários foram elaborados, os quais são: produtividade atingível (com irrigação automática quando requerido) e atual (sequeiro), enquanto para cada cenário foram executadas 12 simulações, sendo uma por mês (início 15 de janeiro e fim em 15 de dezembro), com base nos vinte anos de dados climáticos históricos provenientes da estação automática da Embrapa CNPMF. O modelo simulou satisfatoriamente a produção do feijão caupi nas condições de Cruz das Almas demonstrando sensibilidade às variações climáticas inter e intra-anuais apontando, como mais propícias para a semeadura, as épocas compreendidas entre meados de junho e de julho devido ao fato de apresentarem menor risco de quebra de produtividade.
\end{abstract}

Palavras-chave: modelagem, Vigna unguiculata, DSSAT

\section{Determining the optimum sowing dates for cowpea based on CROPGRO model in Recôncavo of Bahia - Brazil}

\begin{abstract}
The objective of this study was to simulate sowing dates for cowpea (Vigna unguiculata (L.) Walp.) in Cruz das Almas, Recôncavo of Bahia, based on crop model CROPGRO-cowpea. The crop model CROPGRO was calibrated and evaluated based on field experiments data set. Two different scenarios were elaborated, attainable yield (automatic irrigation when required) and observed yield (rainfed) and for each scenario 12 simulations were made, one per month (begin January, 15 and end December, 15), considering 20 years of historic daily weather data set from the weather station at Embrapa CNPMF. The model simulated well the yield for Cruz das Almas, showing sensitivity for the climate variation inter and intra years, being July and June the best month for sowing cowpea due to lesser risk for yield loss.
\end{abstract}

Key words: modeling, Vigna unguiculata, DSSAT

\footnotetext{
1 Instituto Federal de Educação, Ciência e Tecnologia da Bahia, CEP 40300-010, Salvador, BA. Fone: (71) 2102-9536. E-mail: aristides@ifba.edu.br

${ }^{2}$ Laboratório de Irrigação e Fertirrigação/Embrapa Mandioca e Fruticultura Tropical. CEP 44380-000, Cruz das Almas, BA. Fone: (75) 3621-8021. E-mail: macoelho@cnpmf.embrapa.br
}

${ }^{3}$ Embrapa Arroz e Feijão, Rodovia GO 462, km 12, Zona Rural, CEP 75375-000, Santo Antônio de Goiás, GO. E-mail: alexbh@cnpaf.embrapa.br 


\section{INTRODUÇÃO}

A previsão da produtividade é um componente importante no planejamento do processo produtivo de culturas. Os modelos de simulação permitem analisar cenários considerando-se as diversas combinações dos fatores climáticos, edáficos e da cultura, que influenciam na sua produtividade (Dallacort et al., 2006).

De acordo com Marin et al. (2011) e Caron et al. (2007), modelos de crescimento e desenvolvimento de culturas baseados em processos são passíveis de contribuir no monitoramento e na previsão de produtividade e também como auxiliares na compreensão dos mecanismos envolvidos nas respostas da cultura ao ambiente. A este respeito Bastos (1999) cita a utilização de modelos como grande vantagem para obtenção de resultados de forma rápida e com baixo custo, além de poder auxiliar eficientemente o agricultor em um planejamento e manejo agrícola que proporcionem melhores rendas líquidas.

De acordo com Boote et al. (2010) a simulação de sistemas de culturas tem funções importantes na agricultura, tais como a síntese de pesquisa básica e de integração aumentando a compreensão do pesquisador quanto aos aspectos de fisiologia, da genética e das características do solo; aplicações para fins de gestão em que os sistemas de simulação de culturas são utilizados para avaliar os impactos do clima e na gestão da produção, o uso da água, a utilização de nutrientes e sua lixiviação.

Dentre os modelos utilizados disponíveis o DSSAT (Decision Support System for Agrotechnology Transfer) se caracteriza por se tratar de um sistema computacional que inclui um conjunto de modelos de crescimento de culturas e que tem sido aplicado eficientemente em todo o mundo, por pesquisadores, segundo Nassif et al. (2012).

O modelo CROPGRO é genérico para leguminosas e permite a simulação do crescimento e desenvolvimento de diversas culturas (grão-de-bico, feijão, fava, amendoim e soja) incluindo o caupi. Conforme Bastos (1999) o CROPGRO, além de possuir uma base fisiológica consistente permite, ao usuário, calibrar e aplicar o modelo em distintas condições de solo e clima, por meio de modificações nos arquivos .CUL (cultivar) e .ECO (ecótipo) sendo também possível acrescentar outras espécies ao modelo, modificando os coeficientes do arquivo. SPE (espécie).

O objetivo deste trabalho foi simular, com o modelo CROPGRO, épocas de semeadura do feijão caupi no Recôncavo da Bahia, tomando-se como parâmetro a quebra de produtividade visando selecionar as melhores épocas.

\section{Material e Métodos}

O estudo foi realizado no município de Cruz das Almas, Estado da Bahia, numa área experimental da Embrapa Mandioca e Fruticultura, em outubro de 2010, cujas coordenadas geográficas são: $12^{\circ} 40^{\prime} 39^{\prime \prime} \mathrm{S}$ e $39^{\circ} 06^{\prime} 23^{\prime}$ ' W e a altitude é de $225 \mathrm{~m}$. O clima é tropical quente e úmido, com pluviosidade média anual de $1.170 \mathrm{~mm}$, com variações entre 900 e 1300 mm, constituindo-se os meses de março a agosto os mais chuvosos e de setembro a fevereiro, os mais secos. A temperatura média anual é de $24,5^{\circ} \mathrm{C}$ e umidade relativa de $80 \%$.

$\mathrm{O}$ solo da área experimental é classificado como Latossolo Amarelo Distrófico Típico, textura franco argilo-arenosa (Tabela 1).

As correções e adubações foram realizadas de acordo com a análise química do solo (Tabela 2) e baseadas na recomendação para a cultura. Foram realizadas capinas para controle das plantas daninhas e se aplicou formicida à base de sulfuramida.

A cultura utilizada foi o feijão caupi (Vigna unguiculata L. Walp.), cultivar BRS Guariba, resultado do cruzamento da linhagem IT85F-2687 (introduzida do International Institute of Tropical Agriculture - IITA, em Ibadan, Nigéria), com a linhagem TE87-98-8G (BRS Paraguaçu, lançada em 2002, na Bahia), do Programa de Melhoramento da Embrapa Meio-Norte, em Teresina, PI, cujas sementes foram adquiridas na Embrapa Meio Norte, PI. Cinco experimentos foram instalados em campo, em diferentes épocas de semeadura: março de 2007, irrigado; janeiro de 2010, irrigado, quando foram avaliadas respostas aos diferentes níveis de irrigação: $0,25,50,75$ e $100 \%$ da capacidade de campo; maio de 2010, período de sequeiro; outubro de 2010, irrigado; outubro de 2010, sequeiro e em julho de 2011, em sequeiro. $\mathrm{O}$ espaçamento entre covas foi de 0,70 por $0,20 \mathrm{~m}$, sendo semeadas quatro sementes e deixada uma planta por cova, após o desbaste. A Tabela 3 apresenta as características da planta.

As variáveis meteorológicas de entrada utilizadas pelo modelo, correspondentes aos valores diários de temperatura máxima e mínima do $\operatorname{ar}\left({ }^{\circ} \mathrm{C}\right)$ e precipitação pluviométrica $(\mathrm{mm})$, foram obtidas na estação meteorológica da Embrapa de Cruz das Almas, desde 1990 até 2010 para utilização no módulo "SEASONAL".

Por não se dispor de dados de irradiação solar, estimativas deste parâmetro foram feitas utilizando-se a equação de Angstrom (Angstrom, 1924) que, segundo Tubelis \& Nascimento (1980) é uma expressão empírica simples que permite conhecer a distribuição espacial da irradiação solar recebida na superfície terrestre (Eq. 1):

Tabela 1. Análise física do solo da área experimental

\begin{tabular}{|c|c|c|c|c|c|c|c|c|}
\hline \multirow{2}{*}{$\begin{array}{l}\text { Profundidade } \\
\text { (m) }\end{array}$} & Areia & Silte & Argila & Ds & Dp & CC & PM & AD \\
\hline & & $(\%)$ & & \multicolumn{2}{|c|}{$\mathrm{kg} \mathrm{dm}^{-3}$} & \multicolumn{3}{|c|}{$\left(\mathrm{m}^{3} \mathrm{~m}^{-3}\right)$} \\
\hline $0-0,2$ & 73,2 & 8,7 & 18,1 & 1,67 & 2,53 & 0,1761 & 0,0980 & 0,0781 \\
\hline $0,2-0,4$ & 62,9 & 6,8 & 30,3 & 1,66 & 2,50 & 0,1936 & 0,1514 & 0,0422 \\
\hline $0,4-0,7$ & 60,0 & 7,7 & 32,3 & 1,43 & 2,46 & 0,1837 & 0,1320 & 0,0517 \\
\hline $0,7-1,0$ & 53,3 & 8,1 & 38,4 & 1,39 & 2,40 & 0,2039 & 0,1435 & 0,0604 \\
\hline
\end{tabular}

DS - densidade do solo; Dp - densidade das partículas; CC - capacidade de campo; PM - ponto de murcha; AD - água disponível

Tabela 2. Análise química do solo da área dos experimentos de maio e outubro de 2010 e julho de 2011

\begin{tabular}{|c|c|c|c|c|c|c|c|c|c|c|c|c|}
\hline \multirow{2}{*}{$\mathrm{pH}$} & \multirow{2}{*}{$\begin{array}{c}\text { M.O. } \\
\left(g^{\prime} \mathrm{kg}^{-1}\right)\end{array}$} & $\mathbf{P}$ & K & $\mathrm{Ca}+\mathrm{Mg}$ & $\mathrm{Ca}$ & $\mathrm{Mg}$ & Al & $\mathrm{H}+\mathrm{Al}$ & $\mathrm{Na}$ & $S$ & CTC & \multirow{2}{*}{$\begin{array}{c}V \\
(\%)\end{array}$} \\
\hline & & \multicolumn{2}{|c|}{$\left(\mathrm{mg} \mathrm{dm}^{-3}\right)$} & \multicolumn{8}{|c|}{$\left(\mathrm{cmol}_{\mathrm{c}} \mathrm{dm}^{-3}\right)$} & \\
\hline 6,21 & 1,21 & 14 & 47 & 3,6 & 2,2 & 1,4 & 0,05 & 1,37 & 0,12 & 3,84 & 5,21 & 73,70 \\
\hline
\end{tabular}


Tabela 3. Características fenotípicas da cultivar BRS Guariba

\begin{tabular}{ll}
\hline \multicolumn{1}{c}{ Característica da planta } & \multicolumn{1}{c}{ BRS Guariba } \\
Hábito de crescimento & Indeterminado \\
Porte & Semi-ereto \\
Tipo de folha & Globosa \\
Cor da flor & Branca \\
Cor do cálice & Roxa \\
Cor da corola & Branca \\
Cor da vagem imatura & Verde \\
Cor da vagem matura & Roxa \\
Cor da vagem seca & Roxa \\
Comprimento médio da vagem & $17,8 \mathrm{~cm}$ \\
Número médio de grãos por vagem & 12 \\
Nível de inserção das vagens & Acima da folhagem \\
Número de dias para a floração plena & 41 dias \\
Ciclo & $65-70$ dias \\
\hline
\end{tabular}

Fonte: Freire Filho et al. (2011)

$$
R s=\left[a+b\left(\frac{n}{N}\right)\right] R a
$$

em que:

Rs - irradiação solar global recebida na superfície terrestre $\left(\mathrm{MJ} \mathrm{m}^{-2} \mathrm{~d}^{-1}\right)$

$\mathrm{Ra}$ - Irradiação total recebida em uma superfície plana e horizontal na ausência da atmosfera $\left(\mathrm{MJ} \mathrm{m}^{-2} \mathrm{~d}^{-1}\right)$

$\mathrm{n} \quad$ - duração do brilho solar (h)

$\mathrm{N}$ - fotoperíodo

a e b - parâmetros de regressão do modelo, que caracterizam a transmitância atmosférica, respectivamente 0,25 e 0,50

A irradiação solar total (Ra) foi calculada com a Eq. 2:

$\mathrm{Ra}=\frac{24(60)}{\partial} \cdot \mathrm{G}_{\mathrm{SC}} \cdot \mathrm{d}_{\mathrm{r}} \cdot\left[\omega_{\mathrm{s}} \times \operatorname{sen}(\phi) \times \operatorname{sen}(\delta)+\cos (\phi) \times \cos (\delta) \times \operatorname{sen}\left(\omega_{\mathrm{s}}\right)\right]$

em que:

$\mathrm{G}_{\mathrm{SC}}$ - constante solar $\left(0,082 \mathrm{MJ} \mathrm{m}^{-2} \mathrm{~min}^{-1}\right)$

dr - correção devida à variação da distância terra-sol

$\omega \mathrm{s}$ - ângulo horário do nascer ao pôr do sol

$\phi \quad$ - latitude local $(-0,221 \mathrm{rad})$

$\delta \quad$ - declinação solar (radianos)

A correção devida à variação da distância terra-sol foi obtida com a Eq. 3:

$$
\mathrm{d}_{\mathrm{r}}=1+0,033 \times \cos \left(\frac{2 \pi}{365} \mathrm{~J}\right)
$$

sendo "J" o dia Juliano.

O ângulo horário do nascer ao pôr do sol ( $\omega$ s) foi calculado com a Eq. 4:

$$
\omega_{\mathrm{s}}=\operatorname{arcos}[-\tan (\phi) \times \tan (\delta)]
$$

sendo:

$\omega \mathrm{S}$ - ângulo horário do nascer ao pôr do sol

$\phi \quad$ - latitude de Cruz das Almas (-0,221 rad)

$\delta \quad$ - declinação solar

A declinação solar foi calculada por meio da Eq. 5:

$$
\delta=0,409 \times \operatorname{sen}\left(\frac{2 \pi}{365} \mathrm{~J}-1,39\right)
$$

O fotoperíodo $(\mathrm{N})$ foi calculado com a Eq. 6 :

$$
\mathrm{N}=0,1333 \cdot \omega \mathrm{s} \cdot \frac{180}{\pi}
$$

sendo "N" o fotoperíodo (h).

A caracterização dos experimentos em arquivos do tipo. SNX foi feita com as épocas de semeadura em intervalos mensais, iniciando-se em janeiro e findando em dezembro, totalizando 12 tratamentos (Tabela 4).

\begin{tabular}{|c|c|c|}
\hline Parâmetro & Valor & Fonte \\
\hline \multicolumn{3}{|l|}{ A. .CUL (cultivar) } \\
\hline Área foliar específica & $255 \mathrm{~cm}^{2} \mathrm{~g}^{-1}$ & Exp. local \\
\hline Área máxima de trifólio & $40 \mathrm{~cm}^{2}$ & Exp. local \\
\hline Peso máximo do grão & $0,17 \mathrm{~g}$ & Exp. local \\
\hline Duração do período de enchimento do grão & 16 dias & Exp. local \\
\hline Número médio de grãos/vagem & 13,2 & Exp. local \\
\hline $\begin{array}{l}\text { Tempo requerido para atingir o final do } \\
\text { crescimento da vagem }\end{array}$ & 14 dias & Exp. local \\
\hline $\begin{array}{l}\text { Taxa máxima da relação } \\
\text { (grão/(grão + vagem)) }\end{array}$ & 70 & Frota et al. (2008) \\
\hline Fração de proteína nas sementes & 0,291 & Frota et al. (2008) \\
\hline Fração de óleo nos grãos & 0,065 & Frota et al. (2008) \\
\hline \multicolumn{3}{|l|}{ B. .ECO (ecotipo) } \\
\hline Tempo entre semeadura e emergência & 5 dias & Exp. local \\
\hline $\begin{array}{l}\text { Tempo entre a emergência e a primeira } \\
\text { folha verdadeira }\end{array}$ & 5 dias & Exp. local \\
\hline $\begin{array}{l}\text { Tempo entre a maturidade fisiológica e a } \\
\text { colheita }\end{array}$ & 5 dias & Exp. ocal \\
\hline \multicolumn{3}{|l|}{ C. .SPE (espécie) } \\
\hline Coeficiente de extinção de luz (KCAN) & 0,85 & Souza et al. (2011) \\
\hline Coeficiente de temperatura (FNPGT) & 34 a 35 & Bastos (1999) \\
\hline Peso específico da folha & $0,0039 \mathrm{~g} \mathrm{~cm}^{-2}$ & Exp. Local \\
\hline Concentração de N na folha & 4,1 & Frota et al. (2008) \\
\hline
\end{tabular}

Os parâmetros utilizados nos arquivos .CUL, .ECO e .SPE constam nas Tabelas 5A, 5B e 5C, respectivamente.

Tabela 5. Parâmetros calibrados no arquivo .CUL (cultivar) - A, ECO (ecotipo) - B e da SPE (espécie) - C

A quebra de produtividade foi calculada com a Eq. 7:

Tabela 4. Tratamentos utilizados para simulação de épocas de semeadura do feijão caupi

\begin{tabular}{ccccccccccccc}
\hline & \multicolumn{10}{c}{ Tratamentos } \\
\cline { 2 - 11 } & $\mathbf{1}$ & $\mathbf{2}$ & $\mathbf{3}$ & $\mathbf{4}$ & $\mathbf{5}$ & $\mathbf{6}$ & $\mathbf{7}$ & $\mathbf{8}$ & $\mathbf{9}$ & $\mathbf{1 0}$ & $\mathbf{1 1}$ & $\mathbf{1 2}$ \\
Épocas & $15-J a n$ & $15-\mathrm{fev}$ & $15-\mathrm{Mar}$ & $15-\mathrm{abr}$ & $15-\mathrm{mai}$ & $15-\mathrm{Jun}$ & $15-J u l$ & $15-\mathrm{ago}$ & $15-\mathrm{set}$ & $15-\mathrm{out}$ & $15-\mathrm{Nov}$ & $15-\mathrm{dez}$ \\
\hline
\end{tabular}




$$
\mathrm{Q}=\left(1-\frac{\mathrm{P}_{\mathrm{r}}}{\mathrm{P}_{\mathrm{p}}}\right) \times 100
$$

em que:

Q - quebra de produtividade (\%)

Pr - produtividade simulada atual $\left(\mathrm{kg} \mathrm{ha}^{-1}\right)$, que leva em consideração a dependência da cultura às condições climáticas e não há limitações nutricionais

$\mathrm{P}_{\mathrm{p}}$ - produtividade simulada atingível $\left(\mathrm{kg} \mathrm{ha}^{-1}\right)$ cuja cultura não tem limitações hídricas nem nutricionais

As simulações foram realizadas utilizando-se o CROPGRO versão 4.5 (Hoogenboom et al., 1994). Para se obter a produtividade simulada atingível, acionou-se a função "irrigação automática quando requerida". Para se obter a produtividade simulada atual, retirou-se esta função deixando a cultura sujeita às condições de precipitação natural.

\section{Resultados e Discussão}

Na Figura 1 se encontram os valores médios mensais da precipitação pluviométrica em Cruz das Almas, no Recôncavo Baiano, no período de 1990 a 2010. Observam-se variações na quantidade e na distribuição das chuvas ao longo do ano, em que o período mais seco vai de setembro a fevereiro. A precipitação média varia de $54 \mathrm{~mm}$ em dezembro a $158,7 \mathrm{~mm}$ em junho.

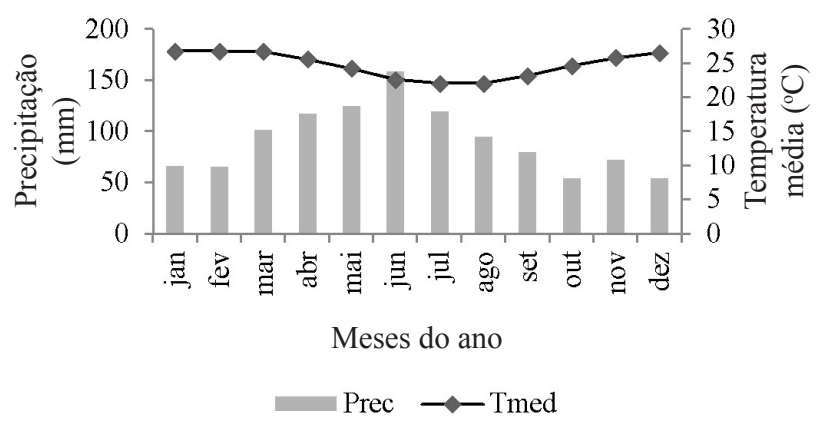

Figura 1. Perfil pluviométrico e variação da temperatura média do ar no período de 1990 a 2010 em Cruz das Almas, BA (Fonte: Estação Meteorológica da Embrapa Mandioca e Fruticultura)

Ainda na mesma figura podem ser analisados os perfis de temperatura média do ar. Os meses de dezembro, janeiro, fevereiro e março, apresentam a temperatura média mais elevada do ano, em torno de $26,8^{\circ} \mathrm{C}$ enquanto a mais baixa ocorre no mês de julho (média de $22,1^{\circ} \mathrm{C}$ ).

A irradiação solar da região é mais elevada nos meses de outubro a março atingindo níveis médios de $21,1 \mathrm{MJ} \mathrm{m}^{-2} \mathrm{~d}^{-1}$, mas atinge níveis mais baixos nos meses de abril a julho, situandose na faixa de $16,4 \mathrm{MJ} \mathrm{m}^{-2} \mathrm{~d}^{-1}$ (Figura 2); portanto, dentro da faixa ideal para o cultivo do feijão que, segundo Ferreira et al. (2011) varia de $13 \mathrm{MJ} \mathrm{m}^{-2} \mathrm{~d}^{-1}$ a $22 \mathrm{MJ} \mathrm{m}^{-2} \mathrm{~d}^{-1}$.

$\mathrm{O}$ balanço hídrico normal para Cruz das Almas com os valores médios de chuva e temperatura compreendidos entre 1990 e 2010, revelou um período de déficit hídrico prolongado compreendido entre os meses de setembro e abril, decorrente da variabilidade anual das chuvas e da capacidade de retenção

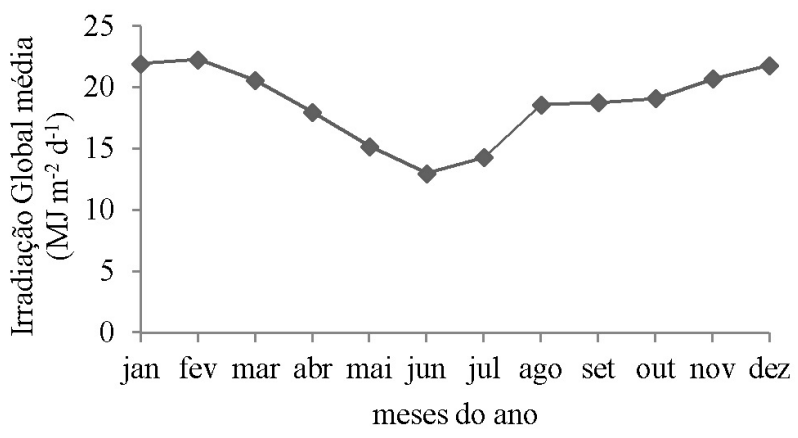

Figura 2. Variação média diária da irradiação solar global no período de 1990 a 2010

de água dos solos predominantes na região, totalizando 374,5 $\mathrm{mm}$. Este déficit corresponde a 32\% do total anual médio da precipitação pluviométrica ocorrida na região, que foi de 1.170 $\mathrm{mm}$. O período de excedente hídrico, que se estende de junho a agosto (Figura 3) é de 108,7 mm. O armazenamento de água no solo atinge $30 \mathrm{~mm}$ nos meses de junho, julho e agosto.

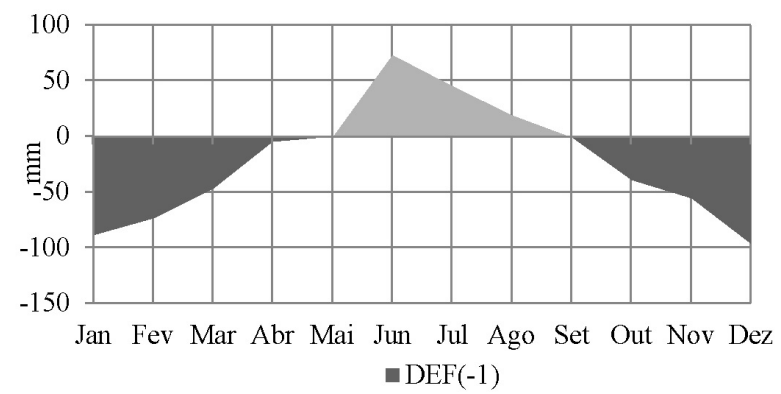

Figura 3. Balanço hídrico normal (mensal), método de Thornthwaite \& Mater (1955), no período de 1990 a 2010

A Tabela 6 apresenta os resultados médios das produtividades simuladas atingíveis. A produtividade média variou de $797,7 \mathrm{~kg} \mathrm{ha}^{-1}$ (15 de abril) a $1.997,7 \mathrm{~kg} \mathrm{ha}^{-1}$ (15 de novembro).

Os coeficientes de variação da produtividade variaram de 8,5 a $17 \%$, sendo considerados baixos, de acordo com Meireles et al. (2003). As maiores produtividades foram obtidas com semeaduras entre 15 de setembro e 15 de dezembro (média de $1.907,1 \mathrm{~kg} \mathrm{ha}^{-1}$ ) e coeficiente de variação médio de $10 \%$.

Tabela 6. Produtividades simuladas atingíveis, média, máximas, mínimas, desvio padrão e coeficiente de variação simulados no período de 1990 a 2010

\begin{tabular}{|c|c|c|c|c|c|c|}
\hline \multirow{2}{*}{ Tratamento } & \multirow{2}{*}{ Semeadura } & Média & Máxima & Mínima & DP & \multirow{2}{*}{$\begin{array}{l}\text { CV } \\
(\%)\end{array}$} \\
\hline & & \multicolumn{4}{|c|}{$\left(k^{\prime} h^{-1}\right)$} & \\
\hline 1 & 15-jan & $1.373,7$ & $1.559,0$ & $1.123,0$ & 121,7 & 8,9 \\
\hline 2 & 15-fev & $1.031,7$ & $1.334,0$ & 813,0 & 137,3 & 13,3 \\
\hline 3 & 15-mar & 857,1 & $1.076,0$ & 663,0 & 109,7 & 12,8 \\
\hline 4 & $15-a b r$ & 797,7 & $1.046,0$ & 586,0 & 113,7 & 14,3 \\
\hline 5 & 15-mai & $1.025,5$ & $1.333,0$ & 706,0 & 161,0 & 15,7 \\
\hline 6 & 15-jun & $1.241,5$ & $1.655,0$ & 917,0 & 161,8 & 13,0 \\
\hline 7 & 15- jul & $1.432,9$ & $1.841,0$ & 898,0 & 243,8 & 17,0 \\
\hline 8 & 15 -ago & $1.637,9$ & $2.139,0$ & $1.046,0$ & 268,7 & 16,4 \\
\hline 9 & 15-set & $1.838,6$ & $2.176,0$ & $1.378,0$ & 204,4 & 11,1 \\
\hline 10 & 15-out & $1.987,0$ & $2.613,0$ & $1.663,0$ & 230,3 & 11,6 \\
\hline 11 & 15-nov & $1.997,7$ & $2.360,0$ & $1.754,0$ & 180,3 & 9,0 \\
\hline 12 & 15-dez & $1.805,1$ & $2.218,0$ & $1.529,0$ & 153,1 & 8,5 \\
\hline
\end{tabular}


Esses resultados são superiores aos encontrados por Freire Filho et al. (2004), que obtiveram produtividades em campo variando de 1.475 a $1.508 \mathrm{~kg} \mathrm{ha}^{-1}$, no Piauí e no Maranhão, respectivamente, com a mesma cultivar e em regime irrigado porém no Recôncavo, Passos et al. (2011) encontraram produtividades inferiores em campo, com médias variando de $142 \mathrm{~kg} \mathrm{ha}^{-1}$ a $1.225 \mathrm{~kg} \mathrm{ha}^{-1}$, com irrigação, em estudo com vinte genótipos de caupi a fim de quantificar o progresso genético para o rendimento de grãos. A distribuição de frequência relativa da produtividade potencial apresentou maior ocorrência entre 797 e $1.037,7 \mathrm{~kg} \mathrm{ha}^{-1}$, em torno de $33 \%$. Levando em conta produtividades acima de $1.517,7 \mathrm{~kg} \mathrm{ha}^{-1}$, elas podem ser encontradas com frequência de $41 \%$ (Figura 4).

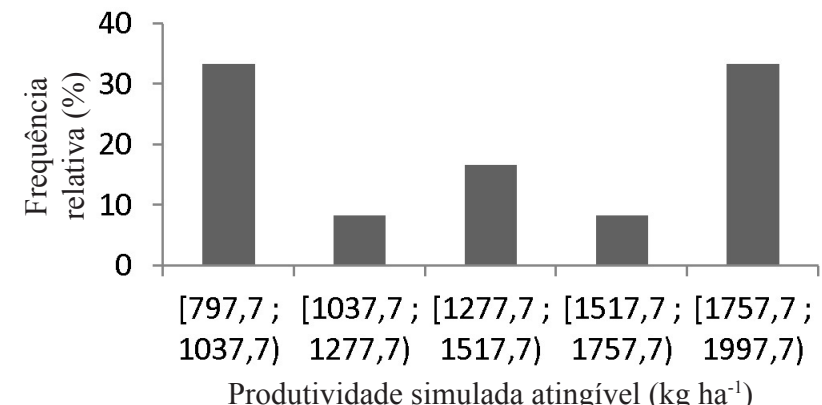

Figura 4. Distribuição de frequência da produtividade potencial para a cultura do feijão caupi

A Tabela 7 apresenta os resultados das simulações atuais (sem irrigação), com suas respectivas produtividades médias, máximas e mínimas e desvios padrões e coeficientes de variação.

Tabela 7. Produtividades atuais médias, máximas, mínimas, desvio padrão e coeficiente de variação simulados no período de 1990 a 2010

\begin{tabular}{|c|c|c|c|c|c|c|}
\hline \multirow{2}{*}{ Tratamento } & \multirow{2}{*}{ Semeadura } & Média & Máxima & Mínima & DP & \multirow{2}{*}{$\begin{array}{l}\text { CV } \\
(\%)\end{array}$} \\
\hline & & \multicolumn{4}{|c|}{$\left(\mathrm{kg} \mathrm{ha}^{-1}\right)$} & \\
\hline 1 & 15-jan & 741,1 & $1.537,0$ & 38,0 & 406,5 & 54,9 \\
\hline 2 & $15-$ fev & 858,1 & $1.511,0$ & 149,0 & 338,8 & 39,5 \\
\hline 3 & 15-mar & 842,8 & $1.076,0$ & 616,0 & 116,9 & 13,9 \\
\hline 4 & $15-a b r$ & 797,7 & $1.046,0$ & 586,0 & 113,7 & 14,3 \\
\hline 5 & 15-mai & $1.025,5$ & $1.333,0$ & 706,0 & 161,0 & 15,7 \\
\hline 6 & 15-jun & $1.231,7$ & $1.655,0$ & 917,0 & 152,5 & 12,4 \\
\hline 7 & 15- jul & $1.270,0$ & $1.611,0$ & 908,0 & 213,6 & 16,8 \\
\hline 8 & 15 -ago & 707,6 & $1.247,0$ & 272,0 & 256,8 & 36,3 \\
\hline 9 & 15-set & 965,8 & $2.321,0$ & 184,0 & 529,4 & 54,8 \\
\hline 10 & 15-out & 601,0 & $1.858,0$ & 165,0 & 413,8 & 68,9 \\
\hline 11 & 15-nov & 869,5 & $2.148,0$ & 108,0 & 561,6 & 64,6 \\
\hline 12 & 15-dez & 910,3 & $1.812,0$ & 101,0 & 539,0 & 59,2 \\
\hline
\end{tabular}

Os resultados mostram uma grande variabilidade, como se previa, uma vez que o regime de chuvas apresenta oscilações penalizando o cultivo sem irrigação. O coeficiente de variação oscilou entre $12,4 \%$ (15 de junho) e $68,9 \%$ (15 de outubro). Por outro lado percebe-se que o modelo CROPGRO é bastante sensível às variações do ambiente. A produtividade variou de $601 \mathrm{~kg} \mathrm{ha}^{-1}$ (15 de outubro) a 1.270,0 $\mathrm{kg} \mathrm{ha}^{-1}$ (15 de julho). As simulações com médias acima de $1.000 \mathrm{~kg} \mathrm{ha}^{-1}$ (15 de maio, 15 de junho e 15 de julho) ocorreram no período das chuvas na região, com baixo coeficiente de variação, média de $13,9 \%$.
Tradicionalmente, o agricultor cultiva o caupi na região, nos meses de julho e agosto. A semeadura em 15 de julho proporciona boa produtividade $\left(1.270,0 \mathrm{~kg} \mathrm{ha}^{-1}\right)$ com baixo coeficiente de variação (16,8\%); já a semeadura de 15 de agosto apresentou produtividade de 707,6 $\mathrm{kg} \mathrm{ha}^{-1}$, com alto desvio padrão (256,8 $\left.\mathrm{kg} \mathrm{ha}^{-1}\right)$ e coeficiente de variação muito elevado $(36,3 \%)$. Para o cultivo em 15 de junho a produtividade esperada é maior $\left(1.231,7 \mathrm{~kg} \mathrm{ha}^{-1}\right)$, com baixo desvio padrão $(152,5 \mathrm{~kg}$ $\left.\mathrm{ha}^{-1}\right)$ e baixo coeficiente de variação $(12,4 \%)$ recomendando-se, portanto, o cultivo entre 15 de junho e 15 de julho.

O menor desvio padrão foi obtido na semeadura de 15 de abril $\left(113,7 \mathrm{~kg} \mathrm{ha}^{-1}\right)$ para um coeficiente de variação de 14,3\% e uma das menores médias de produtividade $\left(797,7 \mathrm{~kg} \mathrm{ha}^{-1}\right)$, porém considerada boa para a média do Nordeste. Na região o dia 19 de março é considerado, tradicionalmente, uma época propícia à semeadura do caupi e do feijão comum (dia de "São José"). Os resultados das simulações para este período na Região do Recôncavo da Bahia apontam uma produtividade esperada de $1.025,5 \mathrm{~kg} \mathrm{ha}^{-1}$, com desvio padrão de $161,0 \mathrm{~kg}$ $\mathrm{ha}^{-1} \mathrm{e}$ um coeficiente de variação considerado baixo $(15,7 \%) \mathrm{de}$ acordo com Meireles et al. (2003). Porém, apesar de estar muito acima da média do Nordeste que, de acordo com Cardoso et al. (1995) é de $300 \mathrm{~kg} \mathrm{ha}^{-1}$, não se recomenda o cultivo do caupi neste período devido à grande probabilidade de ocorrência de doenças como consequência da alta umidade.

A Figura 5 apresenta a distribuição de frequência para as produtividades simuladas atuais com feijão caupi em condição não irrigada, em Cruz das Almas. Percebe-se que a maior frequência (33\%) ocorreu com produtividades entre 734,8 e $868,6 \mathrm{~kg} \mathrm{ha}^{-1}$. A ocorrência de produtividades acima de 1.000 $\mathrm{kg} \mathrm{ha}^{-1}$ é de $25 \%$.

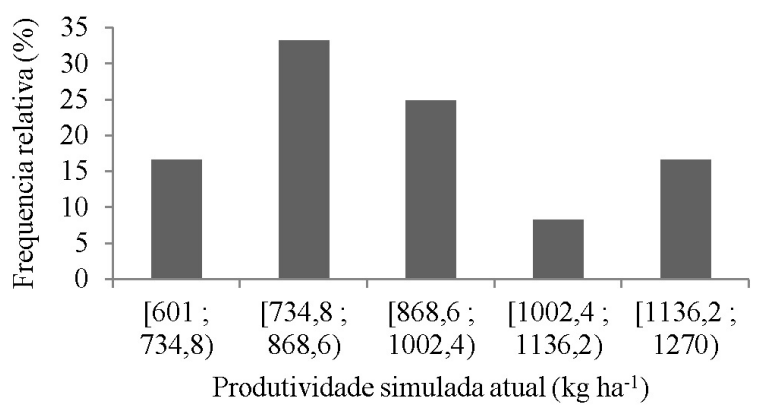

Figura 5. Distribuição de frequência da produtividade real para a cultura do feijão caupi

Analisando as variáveis climáticas utilizadas nas simulações (período de 1990 a 2010), percebe-se que as temperaturas máximas estão dentro da faixa ideal para o caupi porém as temperaturas mínimas registradas entre as semeaduras de maio e agosto foram baixas e, segundo Fancelli \& Dourado Neto (2005), assim como de acordo com Bastos (1999), a temperatura ideal para o caupi deve estar entre 20 e $35^{\circ} \mathrm{C}$. A este respeito Araújo \& Watt (1988) fazem referência às baixas temperaturas noturnas (inferiores a $19{ }^{\circ} \mathrm{C}$ ) como fator responsável pelo atraso na data de floração do caupi e retardamento do seu ciclo, conforme observado nas semeaduras de 15 de maio (86 dias), 15 de junho (88 dias) e 15 de julho (86 dias), apresentadas na Tabela 8 apesar das produtividades terem permanecido acima de $1.000 \mathrm{~kg} \mathrm{ha}^{-1}$ (Tabela 6). 
Tabela 8. Comprimento do ciclo, Irradiação solar média, médias de temperaturas máxima e mínima do ar e precipitação $(\mathrm{P})$, para o período de 1990 a 2010

\begin{tabular}{|c|c|c|c|c|c|}
\hline \multirow{2}{*}{ Tratamento } & \multirow{2}{*}{$\begin{array}{l}\text { Ciclo } \\
\text { (dias) }\end{array}$} & \multirow{2}{*}{$\begin{array}{l}\text { Irrad média } \\
\left(\mathrm{MJ} \mathrm{m}^{-2} \mathrm{~d}^{-1}\right)\end{array}$} & Tmax & Tmin & \multirow{2}{*}{$\underset{(\mathrm{mm})}{\mathbf{P}}$} \\
\hline & & & \multicolumn{2}{|c|}{$\left({ }^{\circ} \mathrm{C}\right)$} & \\
\hline 15-Jan & 70 & 21,7 & 31,6 & 22,1 & 190,0 \\
\hline $15-\mathrm{fev}$ & 71 & 19,8 & 30,7 & 21,9 & 238,8 \\
\hline 15-Mar & 73 & 17,6 & 29,0 & 21,4 & 278,3 \\
\hline $15-a b r$ & 78 & 14,8 & 27,4 & 20,3 & 353,5 \\
\hline 15-mai & 86 & 14,4 & 26,3 & 19,1 & 370,9 \\
\hline 15-Jun & 88 & 16,0 & 26,0 & 18,5 & 316,9 \\
\hline 15-Jul & 86 & 17,9 & 26,7 & 18,7 & 249,8 \\
\hline 15 -ago & 79 & 19,0 & 27,9 & 19,3 & 179,9 \\
\hline 15-set & 76 & 19,8 & 29,5 & 20,4 & 160,7 \\
\hline 15-out & 71 & 20,8 & 30,6 & 21,1 & 153,8 \\
\hline 15-Nov & 70 & 21,6 & 31,4 & 21,7 & 152,9 \\
\hline 15-dez & 69 & 22,1 & 31,7 & 21,9 & 132,5 \\
\hline
\end{tabular}

Comparando as produções simuladas atingíveis com as atuais (Figura 6), percebe-se que de 15 de março a 15 de junho as produtividades são muito próximas sugerindo que, se as temperaturas não influenciaram na produção, assim como a água não foi fator limitante, a irradiação solar pode ter sido a responsável pela limitação da produção, pois a irradiação média neste período foi de $15,7 \mathrm{MJ} \mathrm{m}^{-2} \mathrm{~d}^{-1}$.

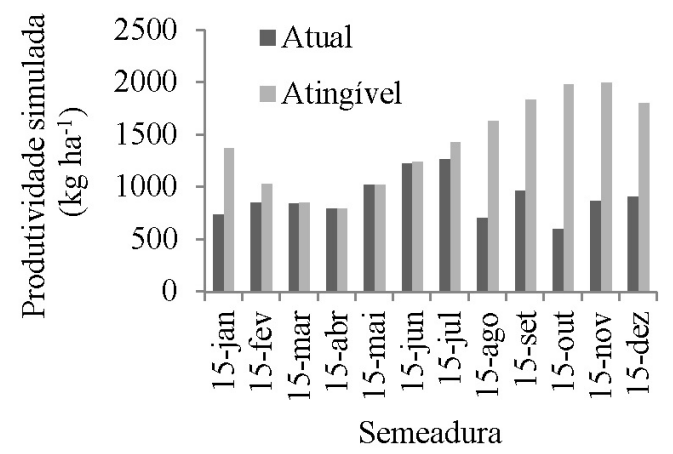

Figura 6. Simulação da produtividade simulada atingível e atual do caupi em Cruz das Almas no período de 1990 a 2010

A Figura 7 apresenta a quebra de produtividade esperada, caso não se utilizasse a irrigação como forma de garantir a produção do caupi em Cruz das Almas.

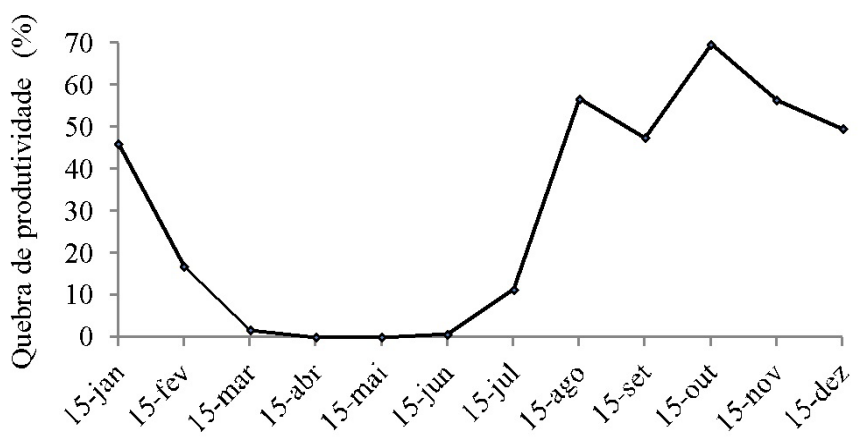

Data de semeadura

Figura 7. Quebra de produtividades esperadas (\%) em função da baixa disponibilidade de água para o caupi
Semeando dentro do período que vai desde 15 de março a 15 de junho, o risco de quebra é muito pequeno (máximo de $1,7 \%$ ) porém, semeadndo-se em março e abril, há risco de excesso de chuva podendo favorecer o aparecimento de doenças e o apodrecimento dos grãos na época da colheita. Em 15 de julho o risco ainda é baixo $(11,4 \%)$ e a partir daí aumenta para $57 \%$ (15 de agosto), reduz para $48 \%$ (15 de setembro) e volta a subir chegando a $70 \%$ em 15 de outubro. Desta forma, os períodos de 15 de junho e 15 de julho provavelmente são os mais recomendados para o cultvio do caupi concordando com EMBRAPA (2003) ao citar que no Nordeste a melhor época de semeadura para as variedades de feijão caupi de ciclo médio (71 a 90 dias), é na metade do período chuvoso de cada região evitando-se que a colheita seja feita em períodos chuvosos, o que aumentaria a probabilidade de apodrecimento de grãos no campo.

Tabela 9. Frequência de quebra relativa de produtividade simulada para o caupi em Cruz das Almas, no período de 1990 a 2010

\begin{tabular}{crrrrr}
\hline \multirow{2}{*}{ Semeadura } & \multicolumn{5}{c}{ Intervalo de quebra de produtividade (\%) } \\
\cline { 2 - 6 } 15-Jan & $\mathbf{0 - 2 0}$ & $\mathbf{2 0 - 4 0}$ & $\mathbf{4 0 - 6 0}$ & $\mathbf{6 0 - 8 0}$ & $\mathbf{8 0 - 1 0 0}$ \\
15-fev & 76,57 & 19,05 & 19,05 & 19,05 & 14,29 \\
15-Mar & 95,24 & 0,00 & 9,52 & 4,76 & 9,52 \\
15-abr & 100,00 & 0,00 & 0,00 & 0,00 & 0,00 \\
15-mai & 100,00 & 0,00 & 0,00 & 0,00 & 0,00 \\
15-Jun & 100,00 & 0,00 & 0,00 & 0,00 & 0,00 \\
15-Jul & 71,43 & 28,57 & 0,00 & 0,00 & 0,00 \\
15-ago & 9,52 & 4,76 & 47,62 & 33,33 & 4,76 \\
15-set & 9,52 & 33,33 & 14,29 & 38,10 & 4,76 \\
15-out & 4,76 & 4,76 & 9,52 & 42,86 & 38,10 \\
15-Nov & 14,29 & 14,29 & 23,81 & 19,05 & 28,57 \\
15-dez & 23,81 & 14,29 & 9,52 & 33,33 & 19,05 \\
\hline
\end{tabular}

Alguns pesquisadores, como Louzada \& Jobim (2011) e Oliveira et al. (2012) comentam quanto à importância da necessidade de irrigação suplementar, mesmo em períodos em que as chuvas são mais frequentes, uma vez que há irregularidades na sua distribuição.

Semeaduras compreendidas entre 15 de fevereiro e 15 de julho apresentam quedas de rendimento inferiores a $20 \%$, ocorrendo em média em $91 \%$ dos casos (Tabela 9) em virtude da maior disponibilidade de água neste período. Já semeaduras entre 15 de agosto e 15 de dezembro, apresentam queda no rendimento acima de 60 em $33 \%$ dos casos evidenciando a importância da irrigação nesta época, uma vez que há grande redução das chuvas. Semeaduras entre 15 de agosto e 15 de outubro proporcionam, em $38 \%$ dos casos, queda de produtividade de 60 a $80 \%$.

\section{Conclusões}

1. O modelo CROPGRO demonstrou sensibilidade às variações das condições ambientais para simulação da produtividade do caupi no Recôncavo.

2. As melhores épocas para a semeadura do feijão caupi no Recôncavo da Bahia estão entre meados de junho e meados de julho.

\section{Literatura Citada}

Angstrom, A. Solar and terrestrial radiation. Quarterly Journal of the Royal Meteorological Society, v.50, p.121-126, 1924. 
Araújo, J. P. P., Watt, E. E. O caupi no Brasil. Brasília: Embrapa CNPAF, 1988. 722p.

Bastos, E. A. Adaptação do modelo CROPGRO para simulação do crescimento e desenvolvimento do feijão caupi (Vigna unguiculata (L.) Walp.) sob diferentes condições hídricas, no Estado do Piauí. Piracicaba: ESALQ/USP, 1999. 91p. Tese Doutorado

Boote, K. J.; Jones, J. W.; Hoogenboom, G.; White, J. W. The role of crop systems simulation in agriculture and environment. International Journal of Agricultural and Environmental Information Systems, v.1, p.41-54, 2010.

Cardoso, M. J.; Andrade Júnior, A. S.; Melo, F. B.; Frota, A. B. Avaliação agroeconômica da produção de sementes de caupi sob irrigação. Teresina: Embrapa CPAMN, 1995. 6p. Comunicado Técnico, 62

Caron, B. O.; Manfrom, P. A.; Lúcio, A. D.; Schmidt, D.; Medeiros, S. L. P.; Bonnecarrère, R. A. G.; Dourado Neto, D. Equações de estimativa da fitomassa aérea da alface. Ciência Rural, v.37, p.1248-1254, 2007.

Dallacort, R.; Freitas, P. S. L.; Faria, R. R.; Gonçalves, A. C. A.; Rezende, R.; Bertonha, A. Utilização do modelo CROPGROsoybean na determinação de melhores épocas de semeadura da cultura da soja, na região de Palotina, Estado do Paraná. Acta Scientiarum: Agronomy, v.28, p.583-589, 2006.

EMBRAPA - Empresa Brasileira de Pesquisa Agropecuária. Embrapa Meio-Norte. Cultivo de feijão-caupi.2003. http:// www.cpamn.embrapa.br/pesquisa/graos/FeijaoCaupi/ referencias.html. 14 Abr. 2012.

Fancelli, A. L.; Dourado Neto, D. Cultura de feijão: Estresse e produtividade. In: Fancelli, A. L.; Dourado Neto, D. (org.). Feijão irrigado: Tecnologia e produção. 1.ed. Piracicaba: Publique, v.1, 2005. p.100-113.

Ferreira, E. P. de B.; Stone, L. F.; Partelli, F. L.; Didonet, A. D. Produtividade do feijoeiro comum influenciada por plantas de cobertura e sistemas de manejo do solo. Revista Brasileira de Engenharia Agrícola e Ambiental, v.15, p.695-701, 2011.

Freire Filho, F. F.; Ribeiro, V. Q.; Rocha, M. M.; Silva, K. J. D. S.; Nogueira, M. S. R.; Rodrigues, E. V. Produção, melhoramento genético e potencialidades do feijão-caupi no Brasil. In: Reunião de Biofortificação, 4, 2011, Teresina. Anais...Teresina: Biofort, 2011. CD-Rom

Freire Filho, F. F.; Ribeiro. V. Q.; Rocha. M. M.; Silva. S. M. de S.; Sittolin, I. M. BRS Guariba nova cultivar de feijão-caupi para a Região Meio-Norte. 2004. http://www. agencia.cnptia.embrapa.br/gestor/feijaocaupi/catalogo/ REC000fltxfsyy02wyiv80kxlb3639xsyys.html. 12 Mai. 2012.
Frota, K. M. G.; Soares, R. A. M.; Arêas, J. A. G. Composição química do feijão caupi (Vigna unguiculata L. Walp), cultivar BRS-Milênio. Ciência e Tecnologia de Alimentos, v.28, p.470-476, 2008.

Hoogenboom, G.; Jones, J. W.; Boote, K. J. Modeling growth, development, and yield of grain legumes using soygro, pnutgro, and beangro: A review. Transactions of the ASAE, v.35, p.2043-2055, 1994.

Louzada, J. A.; Jobim, C. I. P. Demanda de irrigação suplementar e rendimento relativo de grãos de feijão no Rio Grande do Sul. Revista Brasileira de Agrometeorologia, v.16, p.237247, 2011.

Marin, F. R.; Jones, J. W.; Royce, F.; Suguitani, C.; Donzeli, J. L.; Pallone Filho, W. J.; Nassif, D. S. P. Parameterization and evaluation of predictions of DSSAT/CANEGRO for sugarcane Brazilian production systems. Agronomy Journal, v.103, p.100-110, 2011.

Meireles, E. J. L.; Pereira, A. R.; Centelhas, P. C.; Stone, L. F.; Zimmermann, F. J. P. Risco climático de quebra de produtividade da cultura do feijoeiro em Santo Antônio de Goiás, GO. Bragantia, v.62, p.163-171, 2003.

Nassif, D. S. P.; Marin, F. R.; Pallone Filho, W. J.; Resende, R. S.; Pellegrino, G. Q. Parametrização e avaliação do modelo DSSAT/Canegro para variedades brasileiras de cana-de-açúcar. Pesquisa Agropecuária Brasileira, v.47, p.311-318, 2012.

Oliveira, E. C.; Costa, J. M. N.; Paula Júnior, T. J.; Ferreira, W. P. M.; Justino, F. B.; Neves, L. O. The performance of the CROPGRO model for bean (Phaseolus vulgaris L.) yield simulation. Acta Scientiarium, v.34, p.239-246, 2012.

Passos, A. R.; Silva, S. A.; Peixoto, C. P.; Rocha, M. A. C. da; Cruz, E. M. de O. Ganho por seleção direta e indireta em caupi considerando a interação G x E. Revista da FZVA, v.18, p.18-33, 2011.

Souza, R. P.; Machado, E.; Silveira, J. A. G.; Ribeiro, R. V. Fotossíntese e acúmulo de solutos em feijoeiro caupi submetido à salinidade. Pesquisa Agropecuária Brasileira, v.46, p.586-592, 2011.

Thornthwaite, C. W.; Mather, J. R. The water balance. Centerton, N. J. Drexel Institute of Technology - Laboratory of Climatology, 1955. 104p. Publications in Climatology, v.VIII, n.1

Tubelis, A.; Nascimento, F. J. L. Meteorologia descritiva: Fundamentos e aplicações brasileiras. São Paulo: Nobel, 1980. 374p. 